\title{
Gravitational force in an infinite one-dimensional Poisson distribution
}

\author{
A. Gabrielli ${ }^{1,2}$ and M. Joyce ${ }^{3,4}$ \\ ${ }^{1}$ SMC, CNR-INFM, Physics Department, University "Sapienza" of Rome, Piazzale Aldo Moro 2, 00185-Rome, Italy \\ ${ }^{2}$ Istituto dei Sistemi Complessi - CNR, Via dei Taurini 19, 00185-Rome, Italy \\ ${ }^{3}$ Laboratoire de Physique Nucléaire et Hautes Énergies, \\ Université Pierre et Marie Curie - Paris 6, CNRS IN2P3 UMR 7585, \\ 4 Place Jussieu, 75752 Paris Cedex 05, France and \\ ${ }^{4}$ Laboratoire de Physique Théorique de la Matière Condensée, \\ Université Pierre et Marie Curie - Paris 6, CNRS UMR 7600, 4 Place Jussieu, 75752 Paris Cedex 05, France
}

\begin{abstract}
We consider the statistical properties of the gravitational field $F$ in an infinite one-dimensional homogeneous Poisson distribution of particles, using an exponential cut-off of the pair interaction to control and study the divergences which arise. Deriving an exact analytic expression for the probability density function (PDF) $P(F)$, we show that it is badly defined in the limit in which the well known Holtzmark distribution is obtained in the analogous three-dimensional case. A well defined $P(F)$ may, however, be obtained in the infinite range limit by an appropriate renormalization of the coupling strength, giving a Gaussian form. Calculating the spatial correlation properties we show that this latter procedure has a trivial physical meaning. Finally we calculate the PDF and correlation properties of differences of forces (at separate spatial points), which are well defined without any renormalization. We explain that the convergence of these quantities is in fact sufficient to allow a physically meaningful infinite system limit to be defined for the clustering dynamics from Poissonian initial conditions.
\end{abstract}

PACS numbers: 02.50.-r,05.40.-a,61.43.-j

\section{INTRODUCTION}

In attempting to understand better the complex dynamics of classical non-relativistic self-gravitating systems relevant in astrophysics and cosmology, it is of interest to study simplified toy models in one dimension. For systems of a finite number of particles, the evident such model consists of particles on a line interacting by forces independent of their separation (or, equivalently, infinite parallel sheets embedded in three dimensions). This so-called "sheet model" has been quite extensively studied in the literature (see, e.g., [1 7] and references therein) for its interest both as a toy model for gravity in three dimensions and, more generally, as a toy model for systems with long-range interactions. In the study of the formation of structure in the universe in cosmology, the problem may be well approximated, over a large range of length and time scales, by a variant of the simple threedimensional (3D) Newtonian problem: particles belonging to an infinite distribution of particles evolve under equations of motion which are formally identical to those for a finite system, up to simple modifications which take into account the expansion of the universe (see e.g. [8, 9] $)$. A few groups of authors [10 19] have proposed different variants of the simple one-dimensional (1D) sheet model to mimic these equations. We have underlined in a recent paper [20] that a fundamental question about any such model, just as in three dimensions, is whether the gravitational force term - which is simply the infinite sum representing the force exerted by all other particles on the given particle - is well defined in the class of infinite distributions one wishes to study (which will represent the initial conditions for structure formation in cosmology). In [20] we have rigorously shown that, with an appropriate prescription, the infinite sum in one dimension results in a finite and simple expression for the gravitational force acting on particles, in a specific class of infinite point distributions: infinite lattices subjected to a class of stochastic perturbations. In this paper we consider this issue of the definedness of the 1D gravitational force in an infinite and homogeneous Poisson particle distribution, and then the related (but not, as we will discuss, identical) question of whether an infinite system limit for the dynamics of gravitational clustering can be usefully defined in this case.

The approach we use to the question of the definedness of the force follows the seminal work of Chandrasekhar dating back to 1943 [21]: the infinite point distribution is described by a stochastic point process in infinite space, and one considers the statistical properties of the gravitational field at an arbitrary point which is itself then a stochastic quantity. This approach has been adopted and generalized in various cases by ourselves and our collaborators as well as other authors, in the treatment of both gravitational and other forces 22 28]. In his original work Chandrasekhar derived the result for the PDF of the total gravitational field generated by a 3D infinite homogeneous Poisson distribution, which is given by the so-called Holtzmark distribution. It is the generalization of this case to one dimension which is the subject of the present paper. We note that this question has been treated also in a recent paper [29]. The results we present here extend considerably, and allow us to explain the physical meaning of, those presented (for one dimension) in this latter paper.

We recall at the outset that to define the gravitational force in any dimension in any spatially homogeneous in- 
finite mass distribution with non-zero mean density it is necessary (but not necessarily sufficient) to give a prescription for its calculation. This is the case because the gravitational pair force in $d$ dimensions, which decays as $r^{1-d}$ with the separation $r$, gives a force on any particle due to the non-zero mean density which is badly defined. In three dimensions the appropriate prescription (for the cosmological problem) consists in the so-called "Jeans' swindle" (see, e.g., 30, 31]): the contribution of the mean density is removed and only density fluctuations are taken as the source for the gravitational field. As discussed in [32] this "swindle" is a (well-defined) mathematical regularization of the gravitational force acting on particles in the infinite system limit (at constant non-zero mass density), which can be most simply re-stated as the prescription that the force on each particle be calculated by summing symmetrically about it (e.g. in spheres centred on the point). In his calculation of the PDF in three dimensions Chandrasekhar adopted, albeit without explicit discussion, the prescription of summation in such spheres, and other calculations in three dimensions (for other distributions) have done the same. In 24] one of has shown that the adaption of this prescription to one dimension, i.e. summation in a symmetric interval about each point, gives a well defined force PDF, in a class of infinite perturbed lattices, only for pair forces which decrease with separation, but not for the (separation independent) $1 \mathrm{D}$ gravitational force. In [20] we have shown, however, that by adapting a smooth version of this prescription - calculating the total gravitational field as the limit of the sum for an exponentially screened gravitational pair interaction when the screening vanishes continuously - we obtain a well defined PDF also for the gravitational interaction. Thus while in three dimensions the Jeans' swindle can be implemented in various different ways with the same finite results in a wide class of stochastic point-mass distributions, the use of a smooth version of it (rather than a sharp "top-hat" version) has to be preferred in one dimension. This is due to the fact that [20] even in a wide class of very uniform stochastic point process, stochastically perturbed lattices, a top-hat prescription leaves fluctuating and non-converging (i.e. undetermined) boundary contributions which are erased by a smooth regularization. It is this formulation of the Jeans' swindle which we will employ here, although one can, as we will discuss, in fact equally use the "top-hat" prescription and obtain equivalent results in the specific case of a Poisson distribution.

The first result of this paper is an exact expression for the PDF of the screened 1D gravitational force in an infinite Poisson distribution, derived in Sec. III] More specifically we give the exact expression for the cumulants of the PDF. Using this expression we show that the $\mathrm{PDF}$ is, as can be anticipated from a simple qualitative analysis we give in Sec. III badly defined in the limit that the inverse screening length $\mu$ goes to zero. This in three dimensions corresponds to the limit in which instead the well-defined Holtzmark distribution has been obtained by
Chandrasekhar.

In Sec. [IV] we discuss two other ways in which the infinite range limit may be taken, both involving a renormalization of the other system parameters (mean density and coupling strength), and give the (different) asymptotic form of the PDF obtained in each case. The meaning of these renormalisation procedures is explained in Sec. $\nabla$ by means of an exact calculation, again for the screened interaction, of the force-force correlation function. This shows that the non-trivial renormalisation procedures actually give a spatially uniform force (acceleration) field, as in the corresponding limit only the (initially) divergent contribution to the force from particles infinitely far away survives. This means that the dynamics under the renormalised forces corresponds to a trivial (albeit stochastic) rigid translation of all particles, while the spatially varying component of the forces, which would lead to non-trivial relative motions (i.e. in this case, clustering) vanishes.

This discussion leads us naturally to focus on the fact that the spatially non-trivial part of the correlation function is in fact well defined in the simple (unrenormalized) infinite range limit. We show in Sec. VI that this is reflected more generally in the fact that the differences of forces between points at some fixed distance is manifestly well defined and independent of the distribution outside the interval they enclose. We derive an exact expression for the PDF (specifically, again, for its cumulants) of the difference in the (unscreened) gravitational force between two points in space, both without and with the Jeans' swindle (implemented either with a top-hat or the screening prescription). As we are interested in the clustering dynamics manifested in the finite system, i.e. relative motion of particle initially contained in any finite region, we discuss whether the definition of the relative force PDF is sufficient to make the infinite volume limit meaningful. We argue that, if the Jeans' swindle is employed, the limit can indeed be defined.

\section{1D GRAVITATIONAL FIELD IN A POISSON DISTRIBUTION}

Let us consider a homogeneous Poisson particle distribution (i.e. a random particle distribution) [26, 33] on the interval $\left[-L, L^{\prime}\right]$ with average density $n_{0}$. In other words it is characterized by a microscopic density

$$
n(x)=\sum_{i=1}^{N} \delta\left(x-x_{i}\right)
$$

where $x_{i}$ is the position of the $i^{t h}$ particle, and such that

$$
\begin{aligned}
& \langle n(x)\rangle=\lim _{L, L^{\prime} \rightarrow \infty} \frac{N}{L+L^{\prime}}=n_{0}>0 \\
& \left\langle n(x) n\left(x^{\prime}\right)\right\rangle=n_{0}^{2}+n_{0} \delta\left(x-x^{\prime}\right),
\end{aligned}
$$

where $\langle\cdot\rangle$ means the usual ensemble average. The second relation says that there is no correlation between 
the positions of different particles. More generally the joint probability density function (PDF) of the positions of all the particles is simply

$$
p_{N}\left(x_{1}, \ldots, x_{N}\right)=\left(\frac{1}{L+L^{\prime}}\right)^{N}
$$

The $1 \mathrm{D}$ version of the gravitational field generated at $y$ by a particle at $x$ may be written

$$
f(x-y)=g \frac{x-y}{|x-y|}=g \cdot \operatorname{sgn}(x-y) .
$$

The coupling coefficient $g$ gives the intensity of the interaction $^{1}$. The total field at the point $y$ is therefore given by

$$
F(y)=\sum_{i=1}^{N} f\left(x_{i}-y\right)=\int_{-L}^{L^{\prime}} d x n(x) f(x-y) .
$$

We want to study the statistical properties of this quantity in the thermodynamic limit $N, L, L^{\prime} \rightarrow \infty$ with the sole constraint $N /\left(L+L^{\prime}\right) \rightarrow n_{0}$. It is clear that in this limit the integral Eq. (5) is, just as the analogous one in $3 \mathrm{D}$ gravity, ill defined as $n(x) f(x-y)$ is not integrable at large $|x|$ in almost any realization of the Poisson particle system.

It is simple to show by the following simple handwaving argument the result we will find rigorously in the next section: while in 3D the usual "Jeans' swindle" suffices to make the typical force in a Poisson distribution well defined in the infinite system limit, this is not the case in one dimension. We recall that Jeans' swindle consists in considering as the source of the field not the density field $n(x)$, but the density fluctuations $\delta n(x)=\left[n(x)-n_{0}\right]$ from the mean. For any central force, this is equivalent to considering the whole $n(x)$ as the source, but summing symmetrically with respect to the point $y$ where the field is evaluated, e.g. in spheres centered on this point. In the introduction of Chandrasekhar's celebrated derivation 21] of the PDF of the total gravitational field in a 3D homogeneous Poisson particle system, it is this latter procedure which is implicitly followed. In one dimension this corresponds to taking ${ }^{2} L^{\prime}=L+2 y$ in Eq. (5). To see the difference between the two cases (in one and three dimensions), it suffices to estimate, once the Jeans' swindle is adopted, the order of contribution $\Delta F_{>R}$ to Eq. (5) coming from sources at a distance greater than $R$ from the point $\mathbf{y}$. In doing this we approximate the integral in Eq. (5) with a sum over shells defined by the the sequence of radii $R_{n}=2^{n} R$ with integer $n=0,1,2, \ldots$, i.e., radii equally spaced on a logarithmic scale. It is known

\footnotetext{
${ }^{1}$ It is equal to $2 \pi G \Sigma$ when we derive the model from infinite parallel sheets in three dimensions, where $G$ is Newton's constant and $\Sigma$ is the surface mass density of the sheets.

2 This prescription is adopted also in [29].
}

that the typical density fluctuation $\delta n_{V}$ in a given volume $V$ is, for a homogeneous Poisson point process in any spatial dimension, of order $V^{-1 / 2}$. In particular the volume of the $(n+1)^{t h}$ shell is $V_{n}=A\left(2^{n} R\right)^{d}$ where $A$ is a geometrical prefactor depending on $d$ but not on $n$. Therefore we can say that the typical density fluctuation in the $(n+1)^{t h}$ shell is $\delta n\left(R_{n}\right) \simeq\left[A\left(2^{n} R\right)^{d}\right]^{-1 / 2}$. Given that the pair force between two particles at distance $r$ is of order $r^{-d+1}$ in $d$ dimensions, it follows that at sufficiently large $R$ we can approximate Eq. (5) by

$$
\Delta F_{>R} \sim \sum_{n=0}^{\infty} A\left(2^{n} R\right)^{d} \frac{\left[A\left(2^{n} R\right)^{d}\right]^{-1 / 2}}{\left(2^{n} R\right)^{d-1}} \sim C(d) R^{-d / 2+1},
$$

with $C$ a positive constant depending on $d$. Let us start by analyzing Eq. (6) for $d=3$. We can simply verify that $C(d)$ is finite and therefore such a contribution to the total force is finite for any $R$ and vanishes for $R \rightarrow \infty$. Thus, when one sums in spheres about a given point, the typical force on a particle converges. This is the fundamental reason why, once the Jeans' swindle in this form is adapted, the PDF of the gravitational force is well defined (and given, as derived in [21], by the socalled Holtzmark distribution). On the other hand, in $d=1$ one can see that $C(d)=+\infty$, i.e., at any finite $R$ the quantity $\Delta F_{>R}$ is divergent. Moreover the $R$ dependence is pathologically increasing with $R$. This means that, even if one sums symmetrically, the contribution from fluctuations around the mean density due to far away regions always give the dominant diverging contribution to Eq. (5). In the following we will demonstrate this result more formally, showing that the PDF of the total field $F$ calculated in a symmetric window of size $L$ becomes broader and broader as $L$ increases, vanishing for any finite value of $F$ in the limit $L \rightarrow \infty$, i.e., the force $F$ is an ill defined and completely undetermined stochastic quantity even in this symmetric limit.

\section{PDF OF THE REGULARIZED FORCE}

In order to study in a controlled manner the statistical properties of the 1D gravitational force which, as anticipated, may be badly defined, we follow a procedure like that adopted often in the context, notably, of quantum field theory: we introduce a regularization and then study the behaviour of relevant physical quantities in the limit that this regularization is removed. The Jeans' swindle itself can, as discussed in [32], be considered to be such a scheme: in the usual "top-hat" implementation the regularization parameter would then be the size of the symmetric interval (or radius of the sphere in three dimensions) in which one sums. One can equally consider a smooth version in which the symmetric sum is implemented by screening symmetrically the interaction. As discussed in the introduction, it is the latter procedure we adopt here, as we have shown in [20] that this form is, in one dimension, actually preferable to the top-hat form 
because it gives, in a class of more uniform distributions than those considered here, a well defined (and physically meaningful) finite force where the top-hat regularization does not. We thus consider the pair interaction exerted by a particle at $x$ on another at $y$

$$
f_{\mu}(x-y)=g[\operatorname{sgn}(x-y)] e^{-\mu|x-y|},
$$

introducing a cut-off length $\mu^{-1}$ characterising an exponential screening of the "bare" gravitational interaction. We will take the limit $\mu \rightarrow 0^{+}$at the end of our calculation of physically relevant quantities, and specifically, in the next section, to find the existence conditions for the PDF of the total force in this limit.

Given the distribution of $N$ particles in the interval $\left[-L, L^{\prime}\right]$ defined by Eqs. (1), the total field at $y$ is

$$
F_{\mu}(y)=\sum_{i=1}^{N} f_{\mu}\left(x_{i}-y\right)=\int_{-L}^{L^{\prime}} d x n(x) f_{\mu}(x-y) .
$$

Without loss of generality let us now fix $y=0$. Using Eq. (3), the PDF of $F_{\mu}=F_{\mu}(0)$ given $N, L$ and $L^{\prime}$ can be written as $^{3}$

$P\left(F_{\mu} ; N\right)=\int . . \int_{-L^{\prime}}^{L} \prod_{i=1}^{N}\left[\frac{d x_{i}}{L+L^{\prime}}\right] \delta\left[F_{\mu}-\sum_{i=1}^{N} f_{\mu}\left(x_{i}\right)\right]$

By using the identity

$$
\delta(z)=\int_{-\infty}^{+\infty} \frac{d q}{2 \pi} e^{i q z}
$$

we can write

$$
\begin{aligned}
& P\left(F_{\mu} ; N\right)=\int_{-\infty}^{+\infty} \frac{d q}{2 \pi} e^{i q F_{\mu}} \times \\
& \left(\int_{-L^{\prime}}^{L} \frac{d x}{L+L^{\prime}} \exp \left\{-i g q[\operatorname{sgn}(x)] e^{-\mu|x|}\right\}\right)^{N} .
\end{aligned}
$$

In order to take the limit $\left(N, L, L^{\prime}\right) \rightarrow \infty$ with $N /(L+$ $\left.L^{\prime}\right)=n_{0}>0$, we start by writing

$$
\begin{aligned}
& \int_{-L^{\prime}}^{L} \frac{d x}{L+L^{\prime}} \exp \left\{-i g q[\operatorname{sgn}(x)] e^{-\mu|x|}\right\} \\
& \equiv 1-\int_{-L^{\prime}}^{L} \frac{d x}{L+L^{\prime}}\left(1-\exp \left\{-i g q[\operatorname{sgn}(x)] e^{-\mu|x|}\right\}\right) .
\end{aligned}
$$

Since for $\mu>0$ we have

$$
\left|\int_{-\infty}^{+\infty} d x\left(1-\exp \left\{-i g q[\operatorname{sgn}(x)] e^{-\mu|x|}\right\}\right)\right|<+\infty
$$

\footnotetext{
3 Note that, as in a Poisson system there is no correlation of the particle positions, the PDF of the total field at a spatial point is the same whether this point is assumed to be occupied or not. This is a feature specific to Poisson systems, which greatly simply the calculation of the force PDF compared to other cases (see, e.g., discussion in [26]).
}

the above limit of Eq. (11) yields

$$
P\left(F_{\mu}\right)=\int_{-\infty}^{+\infty} \frac{d q}{2 \pi} e^{i q F_{\mu}+\mathcal{G}\left(q ; n_{0}, g, \mu\right)} .
$$

where we have called $P\left(F_{\mu}\right)$ the asymptotic shape of $P\left(F_{\mu} ; N\right)$ for $N, L, L^{\prime} \rightarrow \infty$ with $N /\left(L+L^{\prime}\right)=n_{0}$ and

$$
\mathcal{G}\left(q ; n_{0}, g, \mu\right)=-2 n_{0} \int_{0}^{\infty} d x\left[1-\cos \left(q g e^{-\mu x}\right)\right]
$$

is the cumulant generating function of the stochastic force $F_{\mu}$. The cumulant of $l^{t h}$ order of $F_{\mu}$ is given by

$$
C_{l}\left(n_{0}, g, \mu\right)=\left.i^{l} \frac{d^{l} \mathcal{G}\left(q ; n_{0}, g, \mu\right)}{d q^{l}}\right|_{q=0} \text { for } l \geq 1 .
$$

Note that $C_{1}=\left\langle F_{\mu}\right\rangle$ and $C_{2}=\left\langle F_{\mu}^{2}\right\rangle-\left\langle F_{\mu}\right\rangle^{2}$, and that for a Gaussian variable $\mathcal{G}$ is a quadratic function of $q$. In our case we have that all the odd cumulants vanish by symmetry, while the even ones are

$$
C_{2 l}\left(n_{0}, g, \mu\right)=\frac{n_{0} g^{2 l}}{\mu l} .
$$

\section{RENORMALIZATION SCHEMES}

Let us now analyze the behavior for $\mu \rightarrow 0$, i.e., when the screening length diverges. This is equivalent to applying the Jeans' swindle in the usual way. First of all we see that, as anticipated in the previous section, all the nonzero cumulants diverge $(\sim 1 / \mu)$ and thus the stochastic force $F$ becomes ill defined in the following sense: its PDF $P(F)$ at smaller and smaller $\mu$ becomes broader and broader vanishing point-wise at all $F$. This is analogous to the behavior of the PDF of the sum of $N$ identically distributed and independent random variables with finite variance and zero mean: it becomes broader and broader as $N$ increases and vanishes point-wise at all finite values for $N \rightarrow \infty$. We underline, that this limit $\mu \rightarrow 0$ is also analogous to the one behind Chandrasekhar's calculation 21] in $d=3$ leading to the Holtzmark distribution; and likewise that considered in obtaining a well defined PDF for the $1 \mathrm{D}$ case for a class of perturbed lattices in [20].

It is clear from Eq. (14) that it is possible, however, to converge to a well defined PDF in the limit $\mu \rightarrow 0$ if one appropriately renormalizes also the characteristic scales of the system. There are essentially two different possibilities:

- $n_{0}, \mu \rightarrow 0$ with $n_{0} / \mu=a>0$ and fixed $g>0$ : in this case all the cumulants, and therefore the PDF $P(F)$ are well defined with

$$
C_{2 l}=a \frac{g^{2 l}}{l}>0 \quad \forall l \geq 1 .
$$

This limit is non Gaussian as even cumulants of order larger than two are strictly positive. We call this case the sparse limit (SL) as the particle density vanishes together with $\mu$. 
- $g, \mu \rightarrow 0$ with $g^{2} / \mu=b>0$ and fixed $n_{0}>0$ : in this case also $P(F)$ is well defined. However now $F$ becomes a Gaussian variable for which

$$
C_{2}=b \frac{n_{0}}{2} \text { and } C_{2 l}=0 \text { for } l \geq 2 .
$$

In other words in this limit we have

$$
P(F)=\frac{1}{\sqrt{\pi b n_{0}}} e^{-F^{2} /\left(b n_{0}\right)} .
$$

This limit corresponds to the limit in which a sort of central limit theorem applies to the force $F$. We will refer to it as the weak interaction limit (WIL). This is the limit which is considered in [29].

Thus while the "bare" stochastic force $F$ as defined by the simple sum (5) is ill defined in the infinite volume + infinite force range limit, the two "renormalized" forces $\phi=F \sqrt{\mu / n_{0}}$ and $\psi=F \sqrt{\mu} / g$ are well defined stochastic variables in the same limit. However only the latter is Gaussian.

The first limit is manifestly quite trivial: in the system we consider there are just two characteristic length scales $\lambda_{1}=1 / n_{0}$ (typical interparticle distance) and $\lambda_{2}=1 / \mu$ (range of the interaction). Therefore a system in which these two lengths are substituted by two proportional ones $\lambda_{1}^{\prime}=k \lambda_{1}$ and $\lambda_{2}^{\prime}=k \lambda_{2}$ is just a spatial rescaling of the previous system, which consequently has the same force $F$ statistics $P(F)$. As the latter is non-Gaussian for finite $\mu$ it is likewise so in the asymptotic limit $n_{0}, \mu \rightarrow 0$ with fixed ratio $n_{0} / \mu$. For the other limit, $\mu, g \rightarrow 0$ with fixed $g^{2} / \mu$, this is not the case: rescaling $\mu$ and $g$ to $\mu^{\prime}=k \mu$ and $g^{\prime}=\sqrt{k} g$ does not produce a simple spatial rescaling, i.e., a physically equivalent system.

To understand better the meaning of these different limits it proves instructive to study the spatial correlation properties of the force. This is the subject of the next section.

\section{FIELD-FIELD CORRELATIONS}

Let us consider now, rather than the PDF, the correlation properties of the force field at two distinct spatial points. It is instructive to do so first for the original unregularized $1 \mathrm{D}$ interaction in the finite Poisson system of $N$ particles randomly distributed in the interval $\left[-L, L^{\prime}\right]$. Using Eq. (5), we can write

$$
\langle F(x) F(y)\rangle=g^{2}\left\langle\sum_{i, j}^{1, N} \operatorname{sgn}\left(x_{i}-x\right) \operatorname{sgn}\left(x_{j}-y\right)\right\rangle .
$$

Using Eq. (3) it is straightforward to show that

$$
\begin{aligned}
& \langle F(x) F(y)\rangle=\frac{N}{L^{\prime}+L} g^{2}\left[L^{\prime}+L-2|x-y|\right] \\
& +\frac{N(N-1)}{\left(L^{\prime}+L\right)^{2}} g^{2}\left[\left(L^{\prime}-L\right)^{2}-2(x+y)\left(L^{\prime}-L\right)+4 x y\right]
\end{aligned}
$$

From this formula it is clear that, as for the onepoint properties of the unregularized force, this two-point quantity is ill defined in the limit $N, L, L^{\prime} \rightarrow \infty$ with $N /\left(L^{\prime}+L\right)=n_{0}$. Note that taking the limit symmetrically (i.e. with $L^{\prime}=L$ ) removes the quadratic and linear divergences in the second term, but leaves a linear divergence in the first term. Further, in this case, all spatially dependent terms are in fact finite. This is a crucial point in the discussion of the renormalisation below, which we now formulate for convenience using the smooth regularization procedure used above.

Using instead the pair interaction defined by Eq (7), it is straightforward to obtain, using Eq. (3), and taking the limit $N, L, L^{\prime} \rightarrow \infty$ with $N /\left(L^{\prime}+L\right)=n_{0}$, the result that

$$
G_{\mu}(x-y) \equiv\left\langle F_{\mu}(x) F_{\mu}(y)\right\rangle=n_{0} g^{2}\left(\mu^{-1}-|x-y|\right) e^{-\mu|x-y|}
$$

which, as one would expect, depends only on $|x-y|$. Thus the Jeans' swindle, given by the $\mu \rightarrow 0$ limit, leaves the expression ill-defined. Like Eq. (15) with $L=L^{\prime}$ the term which diverges is space-independent, while the space-dependent part is finite in the same limit.

It is instructive to give the results for the two-point properties also in reciprocal space. The Fourier transform (FT) with respect to $u=(x-y)$ of the two-point correlation function $G_{\mu}(u)$ corresponds to the power spectrum (PS) of the total field, which is thus given by

$$
S_{F}(k ; \mu)=4 n_{0} g^{2} \frac{k^{2}}{\left(k^{2}+\mu^{2}\right)^{2}} .
$$

This result can alternatively be obtained by calculating directly the FT of $F_{\mu}(x)$, as in Appendix A. The integral of the PS of a stochastic field is equal, by definition, to its one point variance, i.e. the integral over $k$ of Eq. (17) is equal to $G_{\mu}(0)=C_{2}\left(n_{0}, g, \mu\right)$. The divergence of $G_{\mu}(0)$ as $\mu \rightarrow 0$ corresponds in $k$-space to the non-integrability of the PS $\left(\propto 1 / k^{2}\right)$ at small $k$, i.e., due to the ill defined large distance correlation behaviour ${ }^{4}$. We note that the result Eq. (17) for the PS of the screened gravitational force field is in fact valid (up to a constant) in any spatial dimension. In three dimensions, however, the unscreened limit $\sim 1 / k^{2}$ is integrable at small $k$ (but non-integrable at large $k$ ). This is an equivalent way of explaining why the Jeans' swindle (formulated using an

\footnotetext{
${ }^{4}$ We note that in any dimension, for the screened interaction, at small $k \ll \mu$ (i.e., at scales much larger than the screening length $\mu^{-1}$ ) the PS $S_{F}(k ; \mu) \sim k^{2}$. This means that $F(x)$ is a so-called superhomogeneous [34], or hyperuniform [35], stochastic field. The main properties of this class of stochastic fields are that $\int_{-\infty}^{+\infty} d x G_{\mu}(x)=0$, and that the fluctuations of the field are subpoissoniann, i.e., in one dimension the normalized fluctuations in a region of size $l$ decrease more rapidly than $l^{-1 / 2}$. For the case of a PS proportional to $k^{2}$ they decay as $\sim l^{-1}$, which is the most rapid possible decay for any proper stochastic process in one dimension
} 
exponential screening) does not work in one dimension while it does in three dimensions. Note, however, that the divergence of the variance alone does not imply in itself that the PDF itself is undefined, which we have shown to be the case in one dimension. Indeed the total force PDF in three dimensions (i.e. the Holtzmark distribution) has infinite variance, due however to the singular behaviour of the 3D pair interaction at vanishing separation and not to the large scale contributions.

Let us now consider again the renormalization schemes introduced in Sect. IV] It is clear that in both cases we obtain

$$
G_{\mu}(x) \rightarrow C_{2} \equiv\left\langle F^{2}\right\rangle_{r e n}
$$

or, equivalently,

$$
S_{F}(k ; \mu) \rightarrow 2 \pi C_{2} \delta(k),
$$

where $\left\langle F^{2}\right\rangle_{\text {ren }}$ stands for the (finite) field variance after the renormalization procedures. These procedures thus keep finite the dominating and diverging contributions to Eq. (15), but at the same time send to zero all other subdominant contributions. In particular this means that they send to zero all terms depending on the spatial argument of the correlation function. In other words, while the regularization+renormalization procedure, in both schemes, makes $F$ well defined as a one-point stochastic quantity, the by-product is to eliminate any space variation of this field. The field which remains is a finite acceleration off-set of the whole system. From a dynamical point of view this corresponds simply to a translation of the whole system, and these renormalisation procedures thus erase all information about the relative motion of particles. Indeed, in the spirit of Mach's principle, all relevant physical information about the force field is lost in this limit: "A particle's inertia is due to some (unfortunately unspecified) interaction of that particle with all the other masses in the universe; the local standards of nonacceleration are determined by some average of the motions of all the masses in the universe, [and] all that matters in mechanics is the relative motion of all the masses" 36.

These observations naturally lead us to consider the statistics of the differences in forces between spatially separated points in the infinite system limit.

\section{RELATIVE FIELD ANALYSIS}

Let us begin again by considering the homogeneous Poisson distribution defined by Eqs. (11) and (3) of $N$ particles in the interval $\left[-L, L^{\prime}\right]$ and interacting by the pair force field (4). Let us fix an arbitrary point, say the origin $x=0$, and consider the difference of the total field at a point $x$ and the origin:

$$
\delta F(x) \equiv F(x)-F(0)=2 g \sum_{i=1}^{N}\left[\theta\left(x_{i}-x\right)-\theta\left(x_{i}\right)\right] .
$$

As the sum is simply, up to a sign depending on $x$, equal to the number of particles in the interval $[0, x), \delta F(x)$ is manifestly independent of the extremes of the interval $\left[-L, L^{\prime}\right]$ and therefore remains the same and well defined in the limit $N, L, L^{\prime} \rightarrow \infty$ with fixed $N /\left(L+L^{\prime}\right)=n_{0}>0$ taken in any arbitrary way. It is thus simple to calculate the one-point PDF of $\delta F(x)$, using the properties of the Poisson distribution:

$$
P(\delta F ; x)=e^{-n_{0}|x|} \sum_{l=0}^{\infty} \frac{\left(n_{0}|x|\right)^{l}}{l !} \delta[\delta F+2 \lg (\operatorname{sgn}(x))]
$$

In other words $\delta F(x)$ can take only (positive or negative depending on the sign of $x$ ) integer multiple values of $2 g$ with a Poisson probability distribution of mean $n_{0}|x|$. Proceeding as for the analysis of $P(F)$ in Sec. III, using the identity Eq. (10), we can derive the characteristic function $\tilde{P}(q ; x)=F T_{\delta F} P(\delta F ; x)$ (where $F T_{\delta F}$ indicates the FT with respect to $\delta F$ ) as

$$
\tilde{P}(q ; x)=\exp [\mathcal{Q}(q ; x)]
$$

where the cumulant generating function $\mathcal{Q}(q ; x)$ of $\delta F(x)$ is given by

$$
\mathcal{Q}(q ; x)=-n_{0}|x|\left(1-e^{i 2 g q \operatorname{sgn}(x)}\right) .
$$

Using the definition Eq. (13) we can obtain the cumulants $\lambda_{j}(x)$ of $\delta F(x)$ for all $j \geq 1$ as

$$
\lambda_{j}(x)=[-2 g \operatorname{sgn}(x)]^{j} n_{0}|x| .
$$

To calculate the same quantity, but now using the "smooth" Jeans' swindle formulated as the $\mu \rightarrow 0$ limit of the screened gravitational interaction Eq. (7), we follow, as in Sec. III, the Chandrasekhar derivation starting directly from the definition of the PDF:

$$
\begin{aligned}
& P_{\mu}(\delta F ; x)=\int . . \int_{-L^{\prime}}^{L} \prod_{i=1}^{N}\left[\frac{d x_{i}}{L+L^{\prime}}\right] \times \\
& \delta\left\{\delta F-g \sum_{i=1}^{N}\left[\operatorname{sgn}\left(x_{i}-x\right) e^{-\mu\left|x_{i}-x\right|}-\operatorname{sgn}\left(x_{i}\right) e^{-\mu\left|x_{i}\right|}\right]\right\} .
\end{aligned}
$$

Following the analogous manipulations and taking the limit $N, L, L^{\prime} \rightarrow \infty$ with fixed $N /\left(L+L^{\prime}\right)=n_{0}>0$ as in Sec. III, we obtain that the cumulant generating functional can be written as

$$
\begin{aligned}
\mathcal{Q}_{\mu}(q ; x)= & -n_{0} \int_{-\infty}^{\infty} d y \times \\
& \left(1-e^{-i q g\left[\operatorname{sgn}(y-x) e^{-\mu|y-x|}-\operatorname{sgn}(y) e^{-\mu|y|}\right]}\right)
\end{aligned}
$$

and thus the cumulants are given as

$$
\begin{aligned}
\lambda_{j}^{\mu}(x)= & (-g)^{j} n_{0} \int_{-\infty}^{\infty} d y \times \\
& {\left[\operatorname{sgn}(y) e^{-\mu|y|}-\operatorname{sgn}(y-x) e^{-\mu|y-x|}\right]^{j} }
\end{aligned}
$$


First of all we notice that

$$
\lambda_{1}^{\mu}(x)=0 \quad \forall \mu>0
$$

It is, furthermore, straightforward to verify that for integer $l$

$$
\lambda_{2 l+1}^{\mu}(x)=-\lambda_{2 l+1}^{\mu}(-x),
$$

while

$$
\lambda_{2 l}^{\mu}(x)=\lambda_{2 l}^{\mu}(-x) .
$$

Finally one can show that for $j \geq 2$

$$
\lim _{\mu \rightarrow 0} \lambda_{j}^{\mu}(x)=\lambda_{j}(x)
$$

i.e., the cumulants of order $j \geq 2$ converge, for $\mu \rightarrow 0^{+}$, to those derived above in Eq. (22) for the case without screening, while the average value $(j=1)$ instead vanishes: the Jeans' swindle simply removes the average density, thus making the average force zero everywhere. The associated generating functional is thus

$$
\mathcal{Q}(q ; x)=-n_{0}|x|\left(1+2 i g q \operatorname{sgn}(x)-e^{i 2 g q \operatorname{sgn}(x)}\right) .
$$

Note that this result may be obtained directly from Eq. (20) by simply replacing $\ell$ inside the delta function by $\ell-n_{0}|x|$, i.e., by simply subtracting by hand the contribution of the mean density $n_{0}$ to the difference in the force $\delta F(x)$. Note that, conversely, we can also obtain the initial result (without the Jeans' swindle) using the second derivation, but putting $\mu=0$ in Eq. (25) before doing the integral. In other words, the two limits, (i) the extremes in the integral in Eq. (25) and (ii) $\mu \rightarrow 0$, cannot be exchanged. The price to pay for this exchange is the uniform contribution coming from the mean density.

It is straightforward also to calculate the two-point correlation functions of $\delta F(x)$. By using again the joint particle positions PDF of Eq. (3) we can simply evaluate the averages and then take the thermodynamic limit which is now well defined, finding

$$
\begin{aligned}
G_{\text {diff }}(x, y) \equiv & \langle\delta F(x) \delta F(y)\rangle= \\
& 2 g^{2} n_{0}\left[|x|+|y|-|x-y|+2 n_{0}|x||y|\right]
\end{aligned}
$$

for the unregularized case (i.e. without Jeans' swindle), and

$$
G_{\text {diff }}(x, y)=2 g^{2} n_{0}[|x|+|y|-|x-y|]
$$

when the Jeans' swindle is used. The latter result is most easily recovered by calculating the correlation function at finite $\mu$ using the result in Eq. (16), and then taking the limit $\mu \rightarrow 0$. The additional quadratic term in Eq. (28) is simply the contribution from the non-zero mean density. The interpretation of the other terms, common to both expressions, is very simple: (i) when $x$ and $y$ have different signs, the two intervals $[0, x)$ and $[y, 0)$ have empty intersection, and, as there is no correlation between the position of particles in a Poisson distribution, the fluctuations in the variables $\delta F(x)$ and $\delta F(y)$ are statistically independent, and the correlation function [the "connected" part for Eq. (28)] is therefore zero; (ii) if instead $x$ and $y$ have the same sign, the segments $[0, x)$ and $[0, y)$ overlap with intersection equal to the shorter of the two segments, and thus there is an non-zero correlation, proportional to the length of this interval.

\section{CONCLUSIONS}

In conclusion let us consider the implications of our results for the question of whether an infinite system limit may be defined for the dynamics of a 1D system of points interacting by the $1 \mathrm{D}$ version of Newtonian gravity, when the initial distribution of these points is Poissonian. More specifically we wish to consider the dynamics of particles described by equations of motion in one dimension given by [20]

$$
\ddot{x}_{i}+2 H \dot{x}_{i}=-\frac{g}{a^{3}} \lim _{\mu \rightarrow 0} \sum_{j \neq i} \operatorname{sgn}\left(x_{i}-x_{j}\right) e^{-\mu\left|x_{i}-x_{j}\right|},
$$

where $a(t)$ is a function describing the expansion of a $3 \mathrm{D}$ universe, and $H=d(\ln a) / d t$ is the corresponding Hubble expansion rate (and the case $a(t)=1$ describes the static universe limit). While in the limit $\mu \rightarrow 0$ these equations are explicitly well defined for a finite number of particles, the question is whether they remain well defined when we consider the usual thermodynamic limit (as defined above: $L, L^{\prime} \rightarrow \infty$ at constant $\left.n_{0}=N / L+L^{\prime}\right)$. The importance of this limit is that it models the case of an infinite universe, which is the application of relevance for these toy models.

We have shown in [20] that this limit may indeed be defined in the case of an infinite array of particles initially displaced off a perfect lattice, for a broad class of such displacements. More specifically we did so by calculating the PDF of the force as defined on the right-hand side of Eq. (30), and showing it to be well defined for this class of distributions. In this paper we have instead shown that the analogous PDF is not well defined for the case of a homogeneous Poisson particle distribution in the same limit, and thus that the infinite system limit is not defined in the same sense.

In our discussion of other possible regularisations of this limit, we have shown that the divergence in the total force arises from the dominant contribution of particles infinitely far away. Because the 1D gravitational force is independent of separation, however, this component does not contribute to the relative force on any two particles at a finite distance. As a result, while the force at any point itself becomes completely undetermined, the difference between forces at two spatially separate points does not. This means that while Eq. (30) is a badly defined equation of motion for each particle $i$, one can 
nevertheless write a well defined equation for the relative displacements $\delta x_{i j} \equiv x_{i}-x_{j}$ of two particles $i$ and $j$ :

$$
\ddot{\delta x_{i j}}+2 H \dot{\delta x_{i j}}=\lim _{\mu \rightarrow 0}\left[F_{\mu}\left(x_{i}\right)-F_{\mu}\left(x_{j}\right)\right]
$$

where

$$
F_{\mu}\left(x_{i}\right)=-\frac{g}{a^{3}} \sum_{k \neq i} \operatorname{sgn}\left(x_{i}-x_{k}\right) e^{-\mu\left|x_{i}-x_{k}\right|} .
$$

Thus, if we consider the evolution from homogeneous Poissonian initial conditions, the position of a particle after any finite time will always depend on $\mu$, and diverge with probability 1 as $\mu \rightarrow 0$. On the other hand, the relative position of any two particles initially at a finite distance will extrapolate to a finite $\mu$-independent value in the same limit. In other words, the clustering dynamics of the system - entirely characterised by the relative positions of particles (e.g. by two or higher point connected correlation properties of the density field) - is well defined. In the spirit of Mach's principle, the diverging absolute displacement, in an infinite system which has intrinsically no centre or preferred point, is not, in any case, of physical relevance.

We have shown the above statements to be true strictly only at the initial time, i.e., the clustering dynamics is well defined in an infinite Poisson particle distribution as we have described. That they remain true as the system evolves away from the initial Poisson distribution can be most easily verified by considering the evolution of the density perturbations in $k$-space. It is well known (and straightforward to show - see e.g. 37]) that, in any spatial dimension, the evolution of a self-gravitating system leads to a $k$-independent amplification of the PS of density fluctuations at small $k$, provided the PS does not vanish faster than $k^{4}$ at $k=0$. The small $k$ (i.e. large scales) scaling behaviour of the PS of a Poisson particle distribution is thus unchanged by evolution. As seen above [cf. discussion around Eq. (17)], it is this behaviour which determines the convergence properties of the force. These properties therefore remain invariant under evolution, guaranteeing that the clustering dynamics remains well defined.

The meaning of this limit is that it corresponds to the clustering dynamics of scales much smaller that $1 / \mu$ for the screened pair interaction, and the limit $\mu \rightarrow 0$ sends this upper cut-off scale to infinity. We remark that this limit exists because the time scale for evolution of clustering at a given spatial scale $x>0$ increases with $x$, i.e. the clustering is what is known as "hierarchical", proceeding from the smallest scale upwards. It is easy to show heuristically, as follows, that this corresponds, in general, to a condition on the scale dependence of the relative forces which is indeed satisfied here. The characteristic time scale $t_{x}$ for evolution of a system on a scale $x$ can be estimated as

$$
t_{x} \sim \sqrt{\frac{x}{|\Delta F(x)|}}
$$

where $|\Delta F(x)|$ is the typical relative force on points at separation $x$. For the case of $1 \mathrm{D}$ gravity in an infinite Poisson distribution, employing the Jeans' swindle as above, we have $\langle[F(x)-F(0)]\rangle=0$ and therefore we take $|\Delta F(x)|^{2}=\left\langle[F(x)-F(0)]^{2}\right\rangle=G_{\text {diff }}(x, x)$. From Eq. (29) it follows then that $t_{x} \sim x^{1 / 4}$. Note, however, that if we do not employ the Jeans swindle, we have instead that the typical force difference on a scale $x$ is $|\langle[F(x)-F(0)]\rangle|=2 g n_{0} x>0$. Therefore now $t_{x}$ is independent of scale, i.e., all scales evolve on the same timescale, which means that the infinite system limit cannot be defined. This is, indeed, the fundamental physical reason for the introduction of the Jeans' swindle: it removes the centre of the system towards which all points otherwise collapse in a time independent of the system size.

Finally let us comment on related results given recently in [29]. This paper derives, using the Jeans' swindle in its usual formulation as a symmetric "top-hat" sum, the PDF of the gravitational force in one dimension, defining it taking the equivalent of the renormalised weak coupling limit we have discussed. A central point in the paper is the observation that in passing from $d=3$ to $d=1$ the statistics of the gravitational force as characterised by the PDF changes from the power-law tailed Holtzmark distribution to the Gaussian form obtained in one dimension Given our results and discussion here, we consider that there is no basis for giving any significance to this fact: the Gaussian PDF in one dimension is not obtained in the analagous limit to that used in three dimensions, and the modified renormalized limit which gives it has only a trivial physical significance as it leads to a spatially trivial force field. Further we note [21, 26] that the "fat" (i.e. non-integrable) power-law tail of the Holtzmark distribution in fact arises from the divergence of the pair interaction at small separations, and has nothing to do with its long range nature. Indeed, even without regulation of the singularity at small separations, other distributions in three dimensions (e.g. "shuffled" lattices with exclusion regions around each particle [26]) have a Gaussian tail in the gravitational force PDF. More generally, in fact, as we will discuss in forthcoming work [38], the long-range nature of a pair interaction does not lead to divergences of all moments of the force PDF of order larger than a typical value, leaving the PDF itself defined even though power law tailed. This is exemplified in the case we have analysed: from Eq. (14) we see that the cumulants of the force field diverge at any order at the same rate as $\mu \rightarrow 0$.

We acknowledge François Sicard for many fruitful discussions in the context of our collaboration on related projects. We also thank Bruno Marcos and Bruce Miller for useful conversations. 


\section{Appendix A: Derivation of the power spectrum of the field $F(x)$}

The power spectrum $S_{F}(k ; \mu)$ given in Eq. (17) can be also derived directly from its definition:

$$
S_{F}(k ; \mu)=\lim _{L, L^{\prime} \rightarrow \infty} \frac{\left\langle\left|\tilde{F}_{\mu}\left(k ; L, L^{\prime}\right)\right|^{2}\right\rangle}{L+L^{\prime}}
$$

where

$$
\tilde{F}_{\mu}\left(k ; L, L^{\prime}\right)=\int_{-L}^{L^{\prime}} d x F_{\mu}(x) e^{-i k x}
$$

with $F_{\mu}(x)$ given by Eqs. (7] 8 ) and $k$ an integer multiple of $2 \pi /\left(L+L^{\prime}\right)$. In order to evaluate $F_{\mu}\left(k ; L, L^{\prime}\right)$ in a simple way it is useful to notice that the pair force (7) can be derived by a simple derivative (and a change of sign) from the pair potential $\phi_{\mu}(x)=-g e^{-\mu|x|} / \mu$ which is a solution of

$$
\frac{d^{2} \phi_{\mu}(x)}{d x^{2}}-\mu^{2} \phi_{\mu}(x)=2 g \delta(x) .
$$

The FT (in $\left.\left[-L, L^{\prime}\right]\right) \tilde{\Phi}\left(k ; L, L^{\prime}\right)$ of the total potential associated with a density field $n(x)$ thus satisfies $\left.\left(k^{2}+\mu^{2}\right) \tilde{\Phi}\left(k ; L, L^{\prime}\right)\right)=-2 g \tilde{n}\left(k ; L, L^{\prime}\right)$. Now substituting $\left|\tilde{F}\left(k ; L, L^{\prime}\right)\right|^{2}=k^{2}\left|\tilde{\Phi}\left(k ; L, L^{\prime}\right)\right|^{2}$ in Eq. (A1), and using the definition of the PS of a Poisson point process

$$
\lim _{L, L^{\prime} \rightarrow \infty} \frac{\left\langle\left|\tilde{n}\left(k ; L, L^{\prime}\right)\right|^{2}\right\rangle}{L+L^{\prime}}=n_{0},
$$

we obtain Eq. (17).
[1] F. Hohl and M. Feix, Astrophys. J., 1471164 (1967).

[2] C. Reidl and B. Miller, Astrophys. J., 371, 260 (1991).

[3] K. R. Yawn and B. N. Miller, Phys. Rev. Lett., 79, 3561 (1997).

[4] G. Severne and M. Luwel, Astrophys. Sp. Sci., 122, 299 (1986).

[5] J.L. Rouet and M. Feix, Phys. Rev. E, 59, 73 (1999).

[6] T. Tsuchiya and N. Gouda, Phys. Rev. E, 61, 73 (2000).

[7] B. Miller, Trans. Th. Stat. Phys. 34, 367 (2005).

[8] J.S. Bagla, Curr. Sci., 88, 10883 (2005).

[9] T. Baertschiger, M. Joyce, A. Gabrielli, F. Sylos Labini, Phys. Rev. E, 75, 021113 (2007).

[10] J. Rouet, M. Feix and M. Navet, Vistas Astron. 33, 357 (1990).

[11] T. Yano and N. Gouda, Astrophy. J. Supp., 118, 267 (1998).

[12] T. Tatekawa and K. Maeda, Astrophy. J., 547, 531 (2001)

[13] E. Aurell, D. Fanelli and P. Muratore-Ginanneschi, Physica D, 148, 272 (2001).

[14] B. Miller and J.L. Rouet, Phys. Rev. E, 65, 056121 (2002)

[15] E. Aurell and D. Fanelli, Astron. Astrophys., 395, 399 (2002).

[16] B. Miller and J.L. Rouet, C. R. Phys.,7, 383 (2006).

[17] P. Valageas, Astron. Astrophys., 450, 445 (2006)

[18] P. Valageas, Phys. Rev. E, 74, 016606 (2006)

[19] B. Miller, J.L. Rouet and E. Le Guirriec, Phys. Rev. E., 76, 036705 (2007).

[20] A. Gabrielli, M. Joyce and F. Sicard, Phys. Rev. E, 80, 041108 (2009).

[21] S. Chandrasekhar, Rev. Mod. Phys., 15, 1 (1943).
[22] A. Gabrielli, F. Sylos Labini, S. Pellegrini, Europhys. Lett., 46, 127 (1999).

[23] A. Gabrielli, A. P. Masucci, F. Sylos Labini, Phys. Rev. E, 69, 031110 (2004).

[24] A. Gabrielli, Phys. Rev. E, 72, 066113 (2005).

[25] A. Gabrielli, T. Baertschiger, M. Joyce, B. Marcos, F. Sylos Labini, Phys. Rev. E, 74, 021110 (2006).

[26] A. Gabrielli, F. Sylos Labini, M. Joyce, and L. Pietronero, Statistical physics for cosmic structures, Springer Verlag Inc. (Berlin, 2004).

[27] A. Del Popolo, and M. Gambera, Astron. \& Astroph., 342, 34 (1998).

[28] J. H.Wesenberg and K. Mølmer, Phys. Rev. Lett., 93, 143903 (2004).

[29] P.H. Chavanis, arXiv:0810.1211v1.

[30] J.H. Jeans, Astronomy and Cosmology, 2nd ed. Cambridge Univ. Press (Cambridge, 1929).

[31] J. Binney and S. Tremaine, Galactic Dynamics, Princeton Series in Astrophysics (Princeton, 1987).

[32] M. K.-H. Kiessling, Adv. Appl. Math., 31, 132 (2003).

[33] J.F.C. Kingman, Poisson processes, Oxford (Oxford, 1993).

[34] A. Gabrielli, M. Joyce, and F. Sylos Labini, Phys. Rev. D, 65, 083523 (2002).

[35] S. Torquato and F. H. Stillinger, Phys. Rev. E, 68, 041113 (2003).

[36] W. Rindler, Essential Relativity, Springler-Verlag, (Berlin, 1977).

[37] P.J.E. Peebles, The Large Scale Structure of the Universe, Princeton Series in Physics, (Princeton, 1980).

[38] A. Gabrielli, M. Joyce, B. Marcos and F. Sicard, in preparation. 